\title{
Perfil dos profissionais atuantes na Gestão em Saúde frente ao novo modelo de reorganização do SUS: A regionalização.
}

\section{Profile of professionals working in Health Care Management with the new public health system reorganization model: Regionalization.}

\section{El perfil de los profesionales que trabajan en la Gestión en Salud frente al nuevo modelo de reorganización del Sistema Único de Salud: La regionalización.}

Dean Carlos Nascimento de MOURA ${ }^{1}$ José Reginaldo PINTO² Antônia Eliana de Araújo ARAGÃO ${ }^{3}$

RESUMO: Este estudo busca analisar o perfil dos profissionais de saúde e sua inserção no novo modelo de reorganização da gestão do SUS por meio do Contrato Organizativo de Ação Pública (COAP), estabelecidos nas regiões de saúde do estado do Ceará. Pesquisa documental, bibliográfica, quantitativa, extraída por fontes de dados secundários, utilizando-se os COAPs de 19 Regiões de Saúde do Estado, produzidos e apresentados no final do ano de 2011 e disponibilizados no site da Secretaria Estadual de Saúde (SESA). O acesso ocorreu durante todo o mês de outubro de 2014. Nesses documentos foram coletados a quantidade de profissionais de saúde, a qualificação desses trabalhadores e os vínculos empregatícios que tinham ao ocupar cargos na gestão dos municípios que compunham as regiões em estudo. Os dados foram apresentados em tabelas, com estatísticas percentuais simples, tabuladas no SPSS versão 20.0. Os achados confirmaram que existe um maior número de profissionais médicos, enfermeiros, auxiliares administrativos e odontólogos distribuídos desigualmente entre as regiões de saúde. Também foi possível visualizar que a maior

1 Biólogo. Especialista em Saúde da Família. Acadêmico do Curso de Enfermagem do Instituto Superior de Teologia Aplicada (Faculdades INTA).

2 Enfermeiro. Mestre em Saúde Coletiva. Docente do Curso de Enfermagem do Instituto Superior de Teologia Aplicada (Faculdades INTA).

3 Enfermeira. Doutora em Enfermagem. Coordenadora do Curso de Enfermagem do Instituto Superior de Teologia Aplicada (Faculdades INTA). 
parte dos trabalhadores eram contratados (57\%), seguido dos terceirizados (30\%). A menor parte tinha vínculo estável, sendo concursados (11,9\%), além dos cedidos $(0,2 \%)$ ou cooperados $(0,9 \%)$. Em relação à qualificação dos empregados, foi demonstrado que houve predominância de cursos de atualização nas regiões de saúde de Acaraú e Sobral. O estudo reflete sobre as condições laborais precárias dos servidores de saúde, com vulnerabilidade de vínculos, distribuição e qualificação irregular por região, que interfere no fortalecimento da regionalização.

Descritores: Sistema Único de Saúde, Gestão em Saúde, Regionalização.

ABSTRACT: This study analyzes the profile of health professionals and their inclusion in the new model of SUS (Public Health System) management reorganization through Organizational Contract of Public Action (COAP), established in health regions of the state of Ceará. Documentary, bibliographical, quantitative research, drawn by secondary data sources, using the COAPs 19 State Health Regions, produced and presented at the end of 2011 and made available on the State Department of Health website (SESA). Access occurred throughout the month of October 2014. These documents demonstrated the amount of health care professionals, the qualification of these workers and work regimes that had to hold positions in the management of counties that constituted the regions studied. The data were presented in tables, simple percentage statistics arranged using SPSS version 20.0. The findings confirm that there were greater number of physicians, nurses, technicians and dentists unevenly distributed between health regions. It was also possible to see that most of the workers were contracted (57\%), followed by a outsourced (30\%). The minority had a stable attachment $(11.9 \%)$, others were assigned $(0.2 \%)$ or cooperative $(0.9 \%)$. Regarding the qualification of employees, it was shown that there was a predominance of refresher courses in health regions Acaraú and Sobral. The study reflects on the poor working conditions of health workers, fragility of employment contracts, distribution and irregular qualification by region, which interferes with the strengthening of regionalization.

Descriptors: Health System, Health Management, Regionalization.

RESUMEN: Este estudio analiza el perfil de los profesionales de salud y su inclusión en el nuevo modelo de la reorganización de la gestión del Sistema Único de Salud a través del Contrato Organizativo de Acción Pública (COAP), establecido en regiones de salud del estado Ceará. Pesquisa documental, bibliográfica, cuantitativa, extraidas por fuentes de datos secundarios, utilizándose los COAPs de 19 Regiones de Salud del Estado, producidos y presentados a finales de 2011 y disponibles en sitio de la Secretaría Estadual de Salud (SESA). El acceso se produjo durante el mes de octubre del 2014. En estos documentos se recogieron la cantidad de profesionales de la salud, la capacitación de estos trabajadores y de los vínculos laborales que tenían al ocupar cargos en la gestión de los municipios que formaban parte de las regiones en estudio. Los datos fueron presentados en tablas, con estadísticas porcentuales simples tabulados mediante el programa SPSS versión 20.0. Los resultados confirman que hay un mayor número de médicos, enfermeras, técnicos y dentistas desigualmente distribuidas entre las regiones de salud. También fue posible visualizar que la mayoría de los trabajadores fueron contratados (57\%) o subcontratados (30\%).

Tempus, actas de saúde colet, Brasília, 10(1), 75-93, mar, 2016.

ISSN 1982-8829 
La menor parte tenía vínculo estable, siendo concursados $(11,9 \%)$, además de los otorgados $(0,2 \%)$ o en cooperativa $(0,9 \%)$. En relación a la calificación de los trabajadores, se demostró que hubo un predominio de cursos de actualización en las regiones de salud de Acaraú y Sobral. El estudio reflexiona sobre las malas condiciones de trabajo de los servidores de salud, con vulnerabilidad de vínculos, distribución y calificación irregular por región, que interfiere con el fortalecimiento de la regionalización.

Descriptores: Sistema Único de Salud, Gestión en Salud, Regionalización.

\section{INTRODUÇÃO}

O Decreto 7.508, de 28 de junho de 2011, veio cumprir o papel de regulamentar a Lei 8.080/90, vinte e um anos após sua publicação, de forma a preencher uma lacuna do arcabouço normativo do Sistema Único de Saúde (SUS). Não é uma inovação, mas reforça a necessidade da gestão do SUS cumprir o que preconiza as legislações emitidas anteriormente a sua criação ${ }^{1}$. Procura organizar as relações interfederativas, mediante a consagração dos colegiados interfederativos tripartite, bipartite e regional, nos quais as decisões são consensuais em razão do compartilhamento da gestão, e definem ainda, as portas de entrada do sistema de saúde, dispondo sobre a hierarquização da complexidade dos seus serviços, a integralidade da assistência, a assistência farmacêutica, metas de desempenho e sua avaliação mediante indicadores de saúde².

O Decreto Presidencial 7.508 de 2011, a Lei Federal 12.466 de 24 de agosto de 2011 e a Lei Complementar 141 de 16 de janeiro de 2012 são instrumentos normativos que propõem a organização do processo de descentralização através da regionalização, sendo fruto de novos contornos ao propor o pressuposto de gestão compartilhada da rede de atenção para sua efetivação ${ }^{3}$. A Lei Complementar $n^{\circ} 141$ regulamenta que o § 3o do art. 198 da Constituição Federal foi elaborada com intuito de regulamentar a Emenda Constitucional 29, estabelecendo os critérios de rateio dos recursos de transferências para a saúde e as normas de fiscalização, avaliação e controle das despesas com saúde nas 3 (três) esferas de governo.

Regionalizar o sistema de saúde é agregar em um sistema cooperativo um conjunto de municípios, impactando positivamente na equidade e na redução dos custos sociais com a atenção à saúde ${ }^{4}$. Além disso, procura estabelecer uma solidariedade entre os municípios circunvizinhos no compartilhamento da resolução de problemas de saúde advindos pela demanda excessiva por procura de atendimento às necessidades de saúde. Dessa forma, essa reorganização dos sistemas locais de saúde, efetivada pela proposta de regionalização, determina uma forma de gestão das cidades mais relacionada à população e seus agravos, vinculados ao seu território ${ }^{5}$.

Uma forma encontrada pelo Ministério da Saúde para efetivar a regionalização do SUS nos estados e municípios foi instituir os Contratos Organizativos da Ação Pública da Saúde (COAP), os quais são acordos de colaboração firmados juridicamente entre os entes da federação com o objetivo de integrar as ações da rede regionalizada e hierarquizada. Nesses contratos, são definidas 
as responsabilidades, os recursos financeiros que serão disponibilizados e as demais ferramentas necessárias para a implementação integrada das ações e serviços de saúde ${ }^{1}$.

O COAP é um instrumento de gestão que define como uma região de saúde pode programar suas ações direcionadas para uma população de forma integrada, tendo a finalidade de tornar mais igualitária à assistência e melhorar o acesso do usuário a oferta de procedimentos e serviços desenvolvidos em um espaço geográfico. As pactuações definidas nesse documento são compartilhadas entre os gestores das Regiões de Saúde, os quais têm prazo de cumprimento determinado por uma ação jurídica normativa, uma vez que os entes federados assinam e confirmam suas responsabilidades e a obrigatoriedade na execução das pactuações.

O COAP contém a seguinte estrutura técnico-jurídica: Parte I: responsabilidade organizativa do SUS, que dispõe sobre os fundamentos do SUS e a atenção à cidadania. Parte II: responsabilidade executiva dos entes federativos. Esta parte dispõe sobre a programação geral do contrato, sendo explicitadas as responsabilidades solidárias dos entes. Parte III: responsabilidade pelo financiamento do contrato que expõem as especificidades locais e estaduais do financiamento. Parte IV: responsabilidade pelo acompanhamento do contrato. Trata-se do monitoramento, avaliação de desempenho da execução do contrato e auditoria ${ }^{1}$.

Os primeiros estados brasileiros a assinarem o COAP foram o Ceará e Mato Grosso do Sul, ainda no ano de 2011. No estado do Ceará, a organização e a tradição dos estados em termos de regionalização de saúde e modelo de gestão dos Consórcios Públicos de Saúde facilitou o processo. O Estado cearense possui 22 Regiões de Saúde e as quatro que formalizaram inicialmente o Contrato foram: Maracanaú, Sobral, Tauá e Brejo Santo ${ }^{6}$.

Apesar de ter passado por dois aditivos e um monitoramento, no estado do Ceará o instrumento ainda continua em vigência até o ano de 2015, mas apresenta desafios que necessitam ser superados pela gestão, como a implantação de alguns serviços pactuados nessa época e que ainda não foram consolidados, dentre eles: o fortalecimento da gestão estratégica e participativa, custeio e financiamentos para a implantação das redes de atenção à saúde (cegonha, urgência e emergência, psicossocial, cuidados às pessoas com deficiência) e o desenvolvimento de uma política de fortalecimentos de vínculos dos profissionais de saúde envolvidos com a gestão dos municípios, em âmbito estadual.

Nesse novo cenário de reorganização do SUS, procurar-se entender como estavam inseridos os profissionais de saúde frente às novas mudanças descritas pelo Decreto 7.508, o COAP e a conformação da regionalização do SUS, ainda no ano da assinatura do instrumento jurídico normativo, que teve a participação dos entes signatários, representados pelos gestores do SUS, especificamente no estado do Ceará.

Nesse contexto, esse estudo busca analisar o perfil dos profissionais que atuavam na gestão 
em saúde e verificar a sua inserção no novo modelo de reorganização do SUS no momento da assinatura dos contratos. Pretende ainda esclarecer quais eram os tipos de vínculos empregatícios dos trabalhadores na época e conhecer que qualificações continham para exercer funções na gestão das Regiões de Saúde do estado.

\section{METODOLOGIA}

Trata-se de uma pesquisa documental, bibliográfica, descritiva, quantitativa, extraída por fonte de dados secundários, utilizando-se os COAPs das Regiões de Saúde do Estado do Ceará, produzidos e apresentados no final do ano de 2011 e disponibilizados no site da Secretaria Estadual de Saúde (SESA).

O estado do Ceará contém 184 municípios, distribuídos em Regiões de Saúde, estando agrupados em cinco Macrorregiões (Fortaleza, Sobral, Cariri, Sertão Central e Litoral Leste/Jaguaribe). Esta divisão é uma resposta ao princípio da descentralização proposto pelo SUS e à necessidade de organização e conformação da rede de atenção à saúde, de acordo com o Decreto 7.508. Essa redefinição, proposta no novo Plano Diretor de Regionalização (PDR), visa traçar um plano de metas para proporcionar uma melhor assistência aos usuários do SUS no estado ${ }^{7,8}$.

O corpus empírico selecionado para análise abrangeu os COAPs das 19 regiões de saúde do estado do ceará, todavia na análise da quantidade de profissionais de saúde existentes em cada documento, foi possível visualizar esse dado apenas nas regiões de Aracati, Camocim, Crato, Icó, Limoeiro e Quixadá. Já na análise de vínculos empregatícios, nas regiões investigadas, foi verificado esse achado apenas em: Aracati, Camocim, Crateús, Juazeiro, Limoeiro e Russas. E ao observar a qualificação dos trabalhadores da gestão dos municípios, percebeu-se que somente as regiões de Acaraú, Aracati, Camocim, Canindé e Sobral apresentaram esse dado em seus COAPs.

Desta forma foram excluídas do estudo os COAPs das regiões de Baturité, Brejo Santo, Cascavel, Caucaia, Maracanaú, Tauá e Tianguá, uma vez que os documentos consultados foram elaborados em diferentes formatações impossibilitando a coleta dos dados para contemplar os objetivos da pesquisa.

Utilizou-se como instrumento de coleta de dados, o mapa da saúde descrito em cada COAP, que se encontrava na parte II deste documento (Responsabilidade Executiva), no item Gestão do Trabalho e da Educação em Saúde.

O acesso ocorreu durante todo o mês de outubro de 2014. Nesse sistema, foi coletada a quantidade de profissionais de saúde, a qualificação desses trabalhadores e os vínculos empregatícios que tinham ao ocupar cargos na gestão dos municípios que compunham as regiões em estudo. $\mathrm{Na}$ análise do perfil dos profissionais de saúde foram consideradas as categorias profissionais para verificar se o total de profissionais eram suficientes para atender a população da região. $O$ tipo de vínculo empregatício foi utilizado para identificar como foi inserido o profissional de saúde no 
mercado de trabalho e se existiam diferenças entre esses vínculos nas regiões. As qualificações ofertadas nas regiões relatadas nos documentos foram analisadas para verificar se atendiam as normatizações definidas no COAP no que tange a implementação da educação permanente e as necessidades de capacitação nesses espaços geográficos.

Nesta perspectiva, a caracterização do perfil a partir das categorias supracitadas propôs uma ferramenta de análise para tomada de decisão dos gestores em relação às necessidades de inserção de diferentes categorias profissionais nas regiões que atendam à população em conformidade com os parâmetros definidos pelo Ministério da Saúde. Já a distribuição e tipo de qualificações ofertadas na região permitiram verificar se existem necessidades não contempladas nos cursos de capacitação para ajudar os gestores no planejamento da oferta de cursos e pactuação de vagas ofertados na região nos anos subseqüentes.

Os dados foram apresentados em tabelas, com estatísticas percentuais simples, tabuladas no SPSS versão 20.0 .

\section{RESULTADOS}

Tabela 1 - Profissionais que atuavam na gestão das Regiões de Saúde do Ceará, 2011.

\begin{tabular}{|c|c|c|c|c|c|c|c|c|c|c|c|c|c|}
\hline \multirow{2}{*}{$\begin{array}{l}\text { PROFISSIONAIS } \\
\text { DE SAÚDE }\end{array}$} & \multicolumn{12}{|c|}{ REGIÕES DE SAÚDE } & \multirow[b]{2}{*}{ TOTAL } \\
\hline & 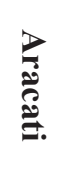 & $\partial^{0}$ & 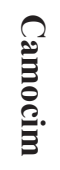 & $0^{\circ}$ & ڤี & $0^{\theta}$ & 8 & $0^{\circ}$ & 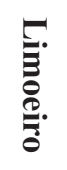 & $0^{e}$ & 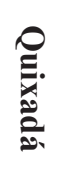 & $\partial^{0}$ & \\
\hline Assistente Social & 9 & 21 & 9 & 21 & 10 & 24 & 5 & 13 & 9 & 21 & - & - & \\
\hline Biólogo & - & - & - & - & 1 & 50 & - & - & - & - & 1 & 50 & 42 \\
\hline Biomédico & 1 & 13 & - & - & 5 & 62 & - & - & - & - & 2 & 25 & \\
\hline Educador Físico & 3 & 9 & 2 & 4 & 4 & 13 & - & - & 4 & 13 & 20 & 61 & \\
\hline Enfermeiro & 72 & 9 & 76 & 10 & 239 & 32 & 33 & 6 & 130 & 17 & 193 & 26 & 35 \\
\hline Farmacêutico & 12 & 9 & 8 & 5 & 47 & 36 & 21 & 16 & 13 & 10 & 30 & 24 & 743 \\
\hline Fisioterapeuta & 12 & 6 & 14 & 7 & 66 & 29 & 24 & 10 & 27 & 12 & 84 & 37 & 131 \\
\hline Fonoaudiólogo & 5 & 10 & 6 & 12 & 11 & 23 & 7 & 15 & 9 & 19 & 10 & 21 & 221 \\
\hline Médicos & 75 & 9 & 62 & 7 & 230 & 28 & 63 & 8 & 62 & 7 & 340 & 41 & 48 \\
\hline Médico Veterinário & 6 & 17 & 1 & 3 & 15 & 42 & - & - & 7 & 19 & 7 & 19 & 832 \\
\hline Nutricionista & 8 & 14 & 8 & 14 & 14 & 25 & 5 & 11 & 8 & 14 & 12 & 22 & 36 \\
\hline Odontólogo & 36 & 9 & 41 & 10 & 115 & 29 & 18 & 5 & 77 & 20 & 104 & 27 & 25 \\
\hline Psicólogo & 9 & 10 & 7 & 8 & 34 & 39 & 7 & 8 & 4 & 6 & 25 & 29 & 391 \\
\hline Terapeuta Ocupacional & 1 & 4 & 3 & 11 & 11 & 41 & - & - & 12 & 44 & - & - & 86 \\
\hline Outros Trabalhadores & 3 & 1 & 227 & 46 & - & - & 215 & 44 & - & - & 43 & 9 & 27 \\
\hline TOTAL & 252 & 8 & 464 & 15 & 802 & 25 & 398 & 13 & 362 & 11 & 871 & 28 & $\begin{array}{r}488 \\
3149\end{array}$ \\
\hline
\end{tabular}

Fonte: Contratos Organizativos de Ação Pública do Ceará, 2011. 
A tabela 1 apresenta o quadro de profissionais existentes em algumas Regiões de Saúde cearenses, onde se pode verificar o maior número de profissionais médicos, enfermeiros, auxiliares administrativos e odontólogos executando atividades laborais nessas regiões.

Houve a existência do maior número de assistentes sociais (24\%), biomédicos (62\%), enfermeiros $(32 \%$ ), farmacêuticos $(36 \%)$, fisioterapeuta $(29 \%)$, fonoaudiólogo $(23 \%)$, médico veterinário (42\%), nutricionista (25\%) e psicólogo (39\%) na Região de Saúde do Crato. Esse fato ocorreu porque Crato possui a maior população (338.742 hab.) das regiões apresentadas nessa tabela.

No entanto, foi encontrado maior número de médicos (41\%), odontólogos (27\%) e educadores físicos (61\%) na Região de Saúde de Quixadá, a qual apresentava uma população de 316.101 habitantes, portanto, menor do que a região de Crato. Esse fato, talvez, se explica devido a proximidade desta região ao centro de formação profissional dessas categorias que está instalado na capital cearense.

Em relação ao profissional biólogo, houve ausência em algumas regiões de saúde avaliadas (Aracati, Camocim, Icó e Limoeiro) descritas nos COAPs. Quanto ao terapeuta ocupacional, sua distribuição foi maior em Icó (44\%), não sendo citada a existência dessa categoria nas regiões de Crato e Limoeiro.

Tabela 2 - Vínculos empregatícios dos profissionais de saúde por municípios nas Regiões de Saúde do Ceará, 2011.

\begin{tabular}{|c|c|c|c|c|c|c|c|c|c|c|c|c|}
\hline \multirow[t]{2}{*}{$\begin{array}{l}\text { REGIÕES } \\
\text { DE SAÚDE }\end{array}$} & \multicolumn{2}{|c|}{ 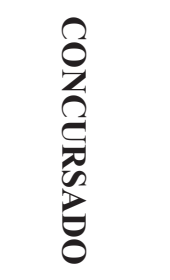 } & \multicolumn{2}{|c|}{ 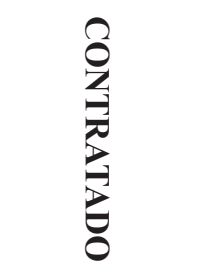 } & \multicolumn{2}{|c|}{ 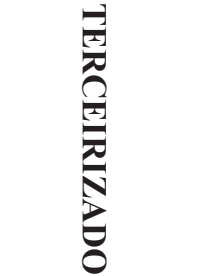 } & \multicolumn{2}{|c|}{ 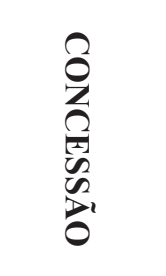 } & \multicolumn{2}{|c|}{$\begin{array}{l}2 \\
8 \\
0 \\
= \\
0 \\
0 \\
0 \\
0\end{array}$} & \multicolumn{2}{|c|}{ TOTAL } \\
\hline & $\mathrm{N}$ & $\%$ & $\mathrm{~N}$ & $\%$ & $\mathrm{~N}$ & $\%$ & $\mathbf{N}$ & $\%$ & $\mathbf{N}$ & $\%$ & $\mathbf{N}$ & $\%$ \\
\hline Aracati & 37 & 0,6 & 223 & 3,5 & - & - & 01 & 0,0 & - & - & 261 & 4,2 \\
\hline Camocim & 264 & 4,3 & 199 & 3,2 & - & - & - & - & - & - & 463 & 7,5 \\
\hline Crateús & - & - & 193 & 3,2 & - & - & - & - & - & - & 193 & 3,2 \\
\hline Juazeiro & 137 & 2,2 & 2713 & 44,3 & 1626 & 26,5 & 02 & 0,0 & 57 & 0,9 & 4535 & 74,0 \\
\hline Limoeiro & 236 & 3,8 & - & - & 196 & 3,2 & 04 & 0,1 & - & - & 436 & 7,2 \\
\hline Russas & 59 & 1,0 & 175 & 2,8 & 01 & 0,0 & 06 & 0,1 & - & - & 241 & 3,9 \\
\hline TOTAL & 733 & 11,9 & 3503 & 57,0 & 1823 & 30,0 & 13 & 0,2 & 57 & 0,9 & 6129 & 100,0 \\
\hline
\end{tabular}

Fonte: Contratos Organizativos de Ação Pública do Ceará, 2011.

A tabela 2 revela que a maioria dos profissionais que trabalhavam nos municípios que compunham as regiões de saúde em evidência eram contratados (57\%), isto é, com vínculo empregatício de caráter temporário, seguido pelos profissionais terceirizados $(30 \%)$. A região onde havia maior número de concursados foi a de Camocim (4,3\%). Juazeiro apresentou-se como a região com maior 
número de contratos $(44,3 \%)$ e de profissionais terceirizados $(26,5 \%)$.

Tabela 3 - Qualificação em gestão em saúde dos profissionais que atuavam nas Regiões de Saúde do Ceará, 2011.

\begin{tabular}{|c|c|c|c|c|c|c|c|c|c|c|c|c|}
\hline \multirow[b]{2}{*}{ CURSOS } & \multicolumn{2}{|c|}{ Acaraú } & \multicolumn{2}{|c|}{ Aracati } & \multicolumn{3}{|c|}{ Camocim } & Canind & \multicolumn{2}{|c|}{ Sobral } & \multicolumn{2}{|c|}{ TOTAL } \\
\hline & 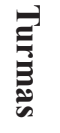 & 胥 & $\vec{E}$ & 胥 & 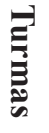 & 胥 & 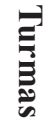 & 吝 & $\bar{E}$ & 胥 & $\vec{E}$ & 胥 \\
\hline $\begin{array}{l}\text { Especialização em Gestão do } \\
\text { SUS }\end{array}$ & 3 & 63 & - & - & 1 & 6 & - & - & 3 & 63 & 7 & 132 \\
\hline $\begin{array}{l}\text { Curso de Aperfeiçoamento } \\
\text { em Regulação, Avaliação e } \\
\text { Auditoria }\end{array}$ & 3 & 63 & - & - & 1 & 6 & - & - & 3 & 63 & 7 & 132 \\
\hline $\begin{array}{l}\text { Técnico em Sistemas de } \\
\text { Informação em Saúde - } \\
\text { formação técnica }\end{array}$ & 1 & 30 & - & - & 1 & 6 & - & - & 1 & 30 & 3 & 66 \\
\hline $\begin{array}{l}\text { Atualização para Secretárias } \\
\text { Executivas dos CMS - } \\
\text { modalidade atualização }\end{array}$ & 1 & 55 & - & - & 1 & 2 & - & - & - & - & 2 & 57 \\
\hline $\begin{array}{l}\text { Atualização sobre o papel da } \\
\text { Coordenação de Atenção Básica }\end{array}$ & 3 & 150 & - & - & 1 & 30 & - & - & 3 & 150 & 7 & 330 \\
\hline $\begin{array}{l}\text { Educação Permanente para o } \\
\text { Controle Social: formação de } \\
\text { operadores da articulação entre } \\
\text { a gestão e o controle social }\end{array}$ & - & - & 1 & 120 & - & - & - & - & - & - & 1 & 120 \\
\hline $\begin{array}{l}\text { Treinamento do Sistema de } \\
\text { Apoio SARGSUS }\end{array}$ & - & - & - & - & - & - & 1 & 15 & - & - & 1 & 15 \\
\hline $\begin{array}{l}\text { Capacitação do Contrato } \\
\text { Organizativo de Ação Pública }\end{array}$ & - & - & - & - & - & - & 1 & 15 & - & - & 1 & 15 \\
\hline TOTAL & 11 & 361 & 1 & 120 & 5 & 50 & 2 & 30 & 10 & 306 & 29 & 867 \\
\hline
\end{tabular}

Fonte: Contratos Organizativos de Ação Pública do Ceará, 2011.

Na tabela 3 há um demonstrativo sobre o perfil de qualificação profissional dos trabalhadores das Regiões de Saúde envolvidas na análise, apresentando-se os cursos, capacitações e treinamentos que a equipe recebeu antes da assinatura dos COAPs. Verifica-se que houve uma maior oferta de vagas para os seguintes cursos: Atualização sobre o papel do Coordenador da Atenção Básica (330 alunos), Curso de Aperfeiçoamento em Regulação, Avaliação e Auditoria (132 alunos) e Especialização em Gestão do SUS (132 vagas). As Regiões de Saúde que tiveram maior número de profissionais capacitados foram Acaraú e Sobral.

Percebeu-se que certos cursos de capacitação não estavam direcionados a todos os profissionais. Talvez esse achado seja relativo à procura e/ou a má distribuição nas pactuações propostas pelo colegiado de gestão nessas Regiões de Saúde responsáveis pela implementação da Educação Permanente em Saúde. 


\section{DISCUSSÃO}

Os primeiros resultados apresentados permitem analisar que é importante explicar as razões pelas quais há um maior número de médicos, enfermeiros, trabalhadores de nível médio e odontólogos nas Regiões de Saúde do Ceará, exibidas na Tabela 1. Esse demonstrativo leva a uma argumentação de que os recursos da saúde, especificamente no SUS, estão diretamente associados à presença desses profissionais, que fazem parte, em sua maioria, das equipes da Estratégia Saúde da Família. No entanto, nota-se uma distribuição irregular desses profissionais por região.

O Conselho Regional de Medicina de São Paulo mostrou em uma pesquisa, que no Brasil, entre a população exclusivamente usuária do SUS, a taxa de médicos ocupados por 1.000 habitantes fica em 1,95. Entretanto, vinte e dois estados brasileiros estão abaixo dessa média. As taxas das capitais reforçam as desigualdades por região: 18 delas têm mais de quatro médicos ocupados por 1.000 habitantes ${ }^{9}$.

Mesmo em número relativo, o Brasil está bem posicionado, dependendo do parâmetro de comparação. Com a densidade de 1.95 médicos/1.000 habitantes, não só se localiza no tercil superior da distribuição, acima da mediana de 1.15 médicos/1.000 habitantes, como também está bem acima da razão do planeta, de 1.4 médicos/1.000 habitantes. Em números absolutos, o país, com 371.788 médicos, apresenta a $5^{\text {a }}$ maior população de médicos do planeta, atrás somente da China (1.905.436), Estados Unidos (793.648), Índia (640.801) e Rússia (614.183). Neste quesito, o Brasil está à frente de 188 países. Os médicos brasileiros representam, sozinhos, 4.05\% da população médica mundial e 19.2\% dos médicos de toda a Região das Américas.

Verifica-se que a distribuição de médicos mais concentrados nas capitais do país é uma regra igualmente válida para as regiões de saúde avaliadas, uma vez que essa distribuição também está mais concentrada em locais onde há maior desenvolvimento econômico nas regiões do Ceará.

De acordo com o Quadro 1, a distribuição dos profissionais de saúde no Estado do Ceará e Região Nordeste em relação ao Brasil, apresenta proporção inferior, especialmente quanto ao profissional médico e enfermeiro, conforme os dados registrados pelo Ministério da Saúde.

Quadro 1 - Parâmetro do número de profissionais de saúde por habitante no Estado do Ceará, Região Nordeste e Brasil, 2010.

\begin{tabular}{|l|c|c|c|}
\hline \multicolumn{1}{|c|}{ Profissões } & Ceará & Nordeste & Brasil \\
\hline Enfermeiros & $1,05 / 1.000 \mathrm{hab}$. & $1,05 / 1.000 \mathrm{hab}$. & $1,81 / 1.000 \mathrm{hab}$. \\
\hline Farmacêuticos & $0,37 / 1.000 \mathrm{hab}$. & $0,37 / 1.000 \mathrm{hab}$. & $0,75 / 1.000 \mathrm{hab}$. \\
\hline Médicos & $1,06 / 1.000 \mathrm{hab}$. & $1,09 / 1.000 \mathrm{hab}$. & $1,86 / 1.000 \mathrm{hab}$. \\
\hline Médicos Veterinários & $0,26 / 1.000 \mathrm{hab}$. & $0,27 / 1.000 \mathrm{hab}$. & $0,58 / 1.000 \mathrm{hab}$. \\
\hline Nutricionistas & $0,12 / 1.000 \mathrm{hab}$. & $0,19 / 1.000 \mathrm{hab}$. & $0,37 / 1.000$ hab. \\
\hline Odontólogos & $0,59 / 1.000$ hab. & $0,65 / 1.000$ hab. & $1,27 / 1.000$ hab. \\
\hline
\end{tabular}

Fonte: DATASUS/MS, $2010^{10}$. 
Confrontando os dados da Tabela 1 com o Quadro 2, percebe-se que a distribuição dos profissionais em todas as Regiões de Saúde do Ceará, por categoria profissional, é desproporcional com o número de habitantes em cada uma delas.

No Brasil, existe uma aparente rigidez no sistema de autorização e controle do exercício profissional médico, por meio de leis que estabelecem monopólios sobre campos de prática, mas não existem políticas voltadas para o controle do estoque das diferentes especialidades ou sua distribuição geográfica e formação, aspectos que têm a ver diretamente com o interesse público. Essa carência de regulação contribui para incentivar a formação de uma proporção elevada de especialistas, para a fragmentação da prática e para a insuficiência na oferta de generalistas. Problemas de infraestrutura também contribuem para a não fixação dos profissionais, como condições inadequadas e até insalubres de unidades de saúde, falta de meios de comunicação e apoio logístico ${ }^{11}$.

Quadro 2 - Distribuição dos profissionais de saúde nas Regiões de Saúde do Ceará, de acordo com os parâmetros nacionais existentes, 2010.

\begin{tabular}{|c|c|c|c|c|c|c|c|c|c|c|c|c|}
\hline \multirow{2}{*}{ 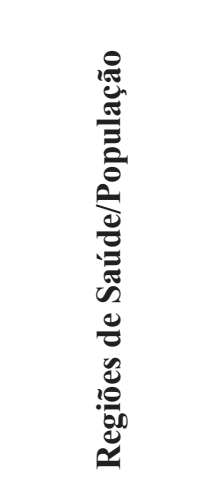 } & \multicolumn{2}{|c|}{ } & \multicolumn{2}{|c|}{ 苞 } & \multicolumn{2}{|c|}{ : } & \multicolumn{2}{|c|}{ 宸 } & \multicolumn{2}{|c|}{ 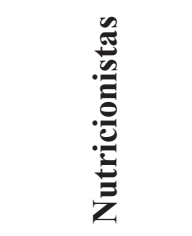 } & \multicolumn{2}{|c|}{ 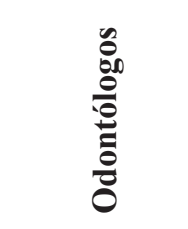 } \\
\hline & 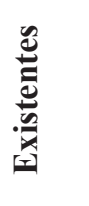 & 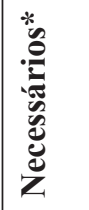 & 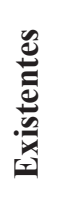 & 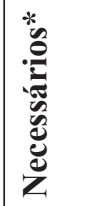 & 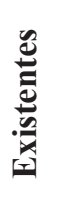 & 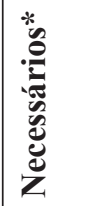 & 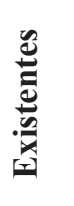 & 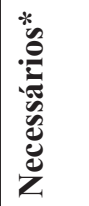 & 苞 & 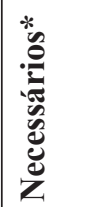 & 总 & 晜 \\
\hline $\begin{array}{c}\text { Aracati } \\
\text { (114.048 hab.) }\end{array}$ & 72 & 206 & 12 & 86 & 75 & 212 & 06 & 66 & 08 & 42 & 36 & 145 \\
\hline $\begin{array}{c}\text { Camocim } \\
(153.682 \text { hab. })\end{array}$ & 76 & 278 & 08 & 115 & 62 & 286 & 01 & 89 & 08 & 57 & 41 & 195 \\
\hline $\begin{array}{c}\text { Crato } \\
\text { (338.742 hab.) }\end{array}$ & 239 & 613 & 47 & 254 & 230 & 630 & 15 & 196 & 14 & 125 & 115 & 430 \\
\hline $\begin{array}{c}\text { Icó } \\
\text { (170.862 hab.) }\end{array}$ & 33 & 309 & 21 & 128 & 63 & 318 & - & 99 & 05 & 63 & 18 & 217 \\
\hline $\begin{array}{c}\text { Limoeiro } \\
\text { (222.195 hab.) }\end{array}$ & 130 & 402 & 13 & 167 & 62 & 413 & 07 & 129 & 08 & 82 & 77 & 282 \\
\hline $\begin{array}{c}\text { Quixadá } \\
\text { (316.101 hab.) }\end{array}$ & 193 & 572 & 30 & 237 & 340 & 588 & 07 & 183 & 12 & 117 & 104 & 401 \\
\hline
\end{tabular}

Fonte: COAP/SESA-CE, 20107.

$\mathrm{Na}$ tentativa de redistribuir igualitariamente os médicos e outros profissionais nas regiões do país, o Ministério da Saúde criou no ano de 2011 o Programa de Valorização do Profissional da Tempus, actas de saúde colet, Brasília, 10(1), 75-93, mar, 2016. 
Atenção Básica (PROVAB) por meio da Secretaria de Gestão do Trabalho e da Educação na Saúde, programa voltado para incentivar a presença de médicos, enfermeiros e cirurgiões-dentistas em municípios com carência de profissionais, em áreas de extrema pobreza e periferias das regiões metropolitanas. Esse programa confere ao participante a possibilidade de Especialização em Saúde da Família e Comunidade por meio de cursos promovidos pela Universidade Aberta do SUS (UNASUS) bem como pontuação para concursos de acesso à residência médica, mediante trabalho em determinadas regiões durante o período mínimo de um ano. Também, em julho de 2013, por meio da Medida Provisória ${ }^{\circ}$ 621, foi instituído o Programa Mais Médico sob a coordenação do Ministério da Saúde ${ }^{12}$.

Similarmente ao PROVAB, o Mais Médicos possui, dentre outros, o objetivo de ampliar a inserção do médico nas unidades de atendimento do SUS, proporcionar maior experiência no campo de prática médica durante seu processo de formação e, ainda, oferecer curso de aperfeiçoamento para seus participantes ${ }^{12}$.

Ao explanar a forma de inserção laboral dos profissionais da gestão do SUS apresentada nos COAPs das Regiões de Saúde avaliadas, verifica-se que a maioria deles não tinha vínculos empregatícios permanentes (Tabela 2). Desta forma, foi possível deparar com uma maioria de profissionais contratados e terceirizados. Esse problema é um ponto de discussão na gestão, ocorrido após a criação da lei 8.080, perdurando na implantação da atenção básica nos municípios, e perpassando pelo Pacto pela Saúde ocorrido em 2006.

Várias conseqüências estão diretamente ligadas a esse problema, dentre estas, pode-se destacar a falta de longitudinalidade na assistência aos usuários, seja na atenção básica, secundária ou terciária, que permite formar uma política de trabalho degradada nas instituições de saúde. Entende-se aqui por longitudinalidade como o estabelecimento da relação terapêutica duradoura entre usuários e profissionais da equipe de saúde, fazendo surgir familiaridade e confiança mútua entre eles, constituindo-se de relações interpessoais, nas quais o profissional foca sua prática na pessoa, não na doença ${ }^{13}$.

Pode ser também conceituado como o acompanhamento dos distintos problemas de saúde por um mesmo profissional, que tece um planejamento de cuidados em longo prazo a um paciente ou comunidade $^{14}$. Isso favorece um comprometimento dos trabalhadores com o cotidiano de vida de cada indivíduo para o qual presta assistência, estreitando uma relação entre família e trabalhador do SUS $^{15}$.

A contratação se torna um problema para a gestão na medida em que pode romper a longitudinalidade da assistência quando os vínculos empregatícios dos trabalhadores forem desatados.

A inserção de novos servidores abruptamente provoca uma fase de readaptação entre usuários 
e profissionais, gerando uma desorganização sistêmica do serviço de saúde, no qual ocorre um rompimento do estabelecimento de vínculos, prejudicando o acesso do usuário ao atendimento do profissional $^{14}$.

Nesse contexto, desfocaliza-se a priorização da qualidade do serviço de saúde por parte dos gestores do SUS em detrimento a uma ampla terceirização do gerenciamento dos serviços de saúde em muitos municípios brasileiros, incentivando a competição dos servidores e a produtividade desenfreada aos moldes do modelo capitalista ${ }^{16}$.

Deve-se pensar que os recursos humanos em serviços de saúde que não tem vínculos fortalecidos, atuam de forma insegura, pois estão constantemente desmotivados e apreensivos com o receio iminente das demissões de cargos, ocasionando-lhes uma desordem psicológica.

Numa outra ótica o empregador utiliza essa fragilidade para coagir o trabalhador a uma maior produtividade, gerando momentos de tensões neste último, refletindo sobre a falta de uma atenção comprometida com o serviço para tentar satisfazer aos ensejos do empregador. Esse fato promove a precarização do trabalhador do SUS, ao gerar um clima de insegurança e instabilidade, promovendo uma cultura de submissão, em que profissionais fragilizados e sem perspectivas de futuro, subordinam-se passivamente aos ditames do poder do capital ${ }^{17}$.

Os dados atuais no âmbito do SUS sobre as modalidades de vínculo empregatício revelam que 800 mil trabalhadores são precarizados, ou seja, cerca de $40 \%$ da força de trabalho está submetida a modalidades contratuais distintas: cooperativas, sistemas de bolsas de trabalho, contratos temporários, pagamento por reconhecimento de dívida, prestação de serviços por meio da Consolidação das Leis Trabalhistas (CLT) etc. ${ }^{18}$.

Uma pesquisa realizada no estado do Ceará, precisamente no Centro de Referência à Saúde do Trabalhador (CEREST), comprova que os profissionais se sujeitam as fragilidades políticas e sociais das relações contemporâneas de precarização do trabalho - terceirizações, aumento da jornada de trabalho, exploração e violência no ambiente laboral; o que compromete sua proposta de integralidade e, consequentemente, a efetividade de suas ações. Acrescem, nesse processo, aspectos geográficos, políticos e culturais próprios da constituição histórica da região que contribuem negativamente para a pouca efetividade das ações de saúde do trabalhador ${ }^{19}$.

Dados de outra pesquisa realizada com servidores públicos do estado da Bahia, que se tratava sobre a experiência de servidores estaduais da saúde em relação à violência no trabalho em saúde, evidenciaram que dos 679 servidores participantes do estudo, 25,9\% dos entrevistados referiram ter sofrido ao menos uma entre as cinco modalidades de violência investigadas (física, verbal, assédio sexual, discriminação e danos contra a propriedade pessoal), sendo a agressão verbal a mais freqüente (19,4\%), atingindo, especialmente, os auxiliares/técnicos de enfermagem (28\%) e os médicos $(23,9 \%)$. Em 66,6\% dos casos, a violência aconteceu por mais de duas vezes ${ }^{20}$. Demonstra-se, dessa forma, que a vulnerabilização dos vínculos laborais pode ocasionar alguns 


\section{$87 / /$}

tipos de violência, como a institucional.

Várias vantagens envolvem o interesse dos gestores do SUS na terceirização e contratação dos profissionais, dentre estas, destacam-se a previsibilidade dos gastos e redução destes encargos trabalhistas, recrutamento, seleção e treinamento de pessoas e imediata reposição de trabalho não adaptado, sem custo adicional. A empresa prestadora de serviço ou mão-de-obra se responsabiliza pelas relações trabalhistas ${ }^{21}$.

Segundo o Ministério da Saúde, o trabalho precário é aquele isento de direitos trabalhistas e de proteção social, como garantia de aposentadoria, gozo de férias anuais, décimo - terceiro salário e às licenças remuneradas de diversos tipos. Estão envolvidos trabalhadores que são contratados diretamente pelo órgão público mediante um vínculo temporário ou informal que se renova sistematicamente; ou ainda, trabalhadores que se incorporam à força de trabalho do setor público por meio de entidades terceirizadas tais como cooperativas e atuam como se fossem autônomos ${ }^{18,22}$.

Verifica-se que na prática de gestão do SUS, dificilmente o profissional contratado goza desses direitos atribuídos pelo Ministério da Saúde, anteriormente mencionados. No Brasil, a precarização na contratação do trabalho recebe o apoio do Estado que cria mecanismos legais para possibilitar a utilização do trabalho informal. A legislação de 1988, por exemplo, assegura três modalidades de flexibilização: redução salarial, compensação de jornada e fixação de turnos ininterruptos de revezamento ${ }^{17}$.

Estudos identificam que as ações implementadas a partir do Governo FHC consolidaram um processo de desinstitucionalização dos direitos preconizados na Constituição Federal de 1988, sobretudo com a privatização dos serviços sociais e com a transferência gradativa da sua gestão para instituições de iniciativa privada ${ }^{18}$.

No entanto, o Conselho Nacional de Secretários Municipais de Saúde (CONASEMS) procurou criar, no ano de 2006, um Comitê Nacional de Desprecarização do trabalho no SUS posicionandose contra qualquer vínculo, seja na forma direta ou indireta, que não assegurasse ao trabalhador os seus direitos trabalhistas e previdenciários. Além disso, defendia uma gestão local que se democratizasse nas relações de trabalho, possibilitando que o trabalhador de saúde fosse sujeito e protagonista na história de construção do SUS. Procurou estimular o processo de sindicalização e a organização do trabalhador no seu ambiente de trabalho e, sobretudo, a criação de espaços de negociação para o debate de suas reivindicações ${ }^{23}$.

Apesar desse intuito, demonstra-se claramente que o quadro de funcionários do SUS descritos nos COAPs, no estado do Ceará, remete a uma realidade distorcida daquela apregoada pelo Ministério da Saúde, ainda no ano de 2011. Nos municípios e na Secretaria Estadual de Saúde desse estado ainda prevalece profissionais isentos de planos de cargos, carreiras e salários de forma a modificar essa realidade. Mesmo assim esta foi uma pauta de discussão entre os gestores do SUS, 
na época da assinatura do documento, presente na cláusula terceira da Parte I do COAP, que trata das Responsabilidades Organizativas.

Destarte, os gestores da saúde necessitam desenvolver uma nova política pública visando à conscientização de que a contratação gera investimentos e gastos instáveis com a inserção de profissionais nessa modalidade de vinculação de recursos humanos, uma vez que se oferecem capacitações, treinamentos e cursos para esses servidores, que por pretextos banais podem ser desvinculados dos serviços por alguma instabilidade gerencial nos municípios, ocasionando uma despesa orçamentária para requalificação de novos profissionais quando ocuparem novos cargos.

Outro ponto de discussão que deve ser conversado ao se expor a Tabela 3, refere-se ao fato da distribuição das capacitações ofertadas aos profissionais que exerciam atividades laborais nos municípios das Regiões de Saúde apresentadas. No tocante a esse fato, percebe-se que duas regiões de saúde se destacaram na oferta de cursos. No entanto, a maior parte dessas qualificações não estava em conexão ao novo processo de reorganização do sistema de saúde proposto (regionalização). Isso porque o COAP anunciava a conformação das Redes de Atenção à Saúde. Nesse caso os profissionais deveriam estar qualificados para atuarem visando à estruturação dessas redes para fortalecer assistencialmente ainda mais as regiões.

Para vários estudiosos sobre qualificação em saúde, as micro intervenções educacionais são ineficazes e apresentam altos custos diretos e indiretos pelo tempo subtraído constantemente do trabalho dos profissionais nas unidades ${ }^{24}$.

As práticas de Educação Permanente em Saúde (EPS) realizadas expressam a verticalidade e a centralidade em conhecimentos específicos. Fica explícito que as necessidades reais dos profissionais não estão presentes no desenvolvimento da EPS. Essa se mostra em formas autoritárias e mecanizadas de educação, onde o saber e a vivência do profissional não são valorizados, tornando-a frágil para ser efetivada no cotidiano das práticas profissionais ${ }^{25}$.

A educação permanente deve apresentar um cenário que envolve a metodologia da problematização, uma equipe com profissionais de diversas áreas de atuação, com ênfase nas situações-problema das práticas cotidianas, possibilitando reflexões críticas e articulando soluções estratégicas em coletivo, e está inserida no desenvolvimento e na consolidação do SUS ${ }^{5,26}$.

Devemos lembrar que a Lei Orgânica da Saúde determinava em seu artigo 14 que deveriam ser criadas Comissões Permanentes de integração entre os serviços de saúde e as instituições de ensino profissional e superior, indicando que cada uma dessas Comissões tivesse por finalidade propor prioridades, métodos e estratégias para a formação e educação continuada dos recursos humanos do SUS na esfera correspondente, assim como em relação à pesquisa e à cooperação técnica entre essas instituições ${ }^{27}$.

Alguns autores criticam que apesar da criação da Secretaria de Gestão do Trabalho e da Tempus, actas de saúde colet, Brasília, 10(1), 75-93, mar, 2016. 
Educação na Saúde (SGTES) do Ministério da Saúde, no ano de 2003, tenha inserido o trabalho e a formação profissional na agenda de formulação das políticas públicas de saúde no país, as questões relacionadas ao trabalho e aos trabalhadores permanecem como um dos maiores problemas a ser enfrentado no âmbito do SUS ${ }^{28}$.

Em relação à conformação da qualificação dos servidores estaduais e municipais no Ceará, pode-se comentar que para operacionalizar a Política Estadual de Educação Permanente em Saúde (PEEPS), que foi alinhada à Política Nacional de Educação Permanente em Saúde (PNEPS), a SESA adotou a classificação expressa no Plano Diretor de Regionalização da Assistência à Saúde do Estado que o subdivide em três Macrorregiões de Saúde, designadas: Fortaleza (99 municípios articulados em 14 Coordenadorias Regionais de Saúde - CRES), Sobral (55 municípios em 05 Coordenadorias Regionais de Saúde) e Cariri (29 municípios em 03 CRES), orientadas a constituir as Comissões Permanentes de Integração Ensino-Serviço (CIES) regional e um Colegiado de Gestão Regional (CGR), hoje Comissão Intergestora Regional (CIR). Para demarcar o papel regulador do estado, criou-se ainda a CIES Estadual, configurando-se enquanto um Fórum Estadual das CIES, sendo um espaço de troca de experiências e decisões relativas às ações de EPS entre as 03 CIES macrorregionais ${ }^{29}$.

As CIR devem ter o apoio das Comissões de Integração Ensino-Serviço (CIES), sendo estas instâncias intersetoriais e interinstitucionais permanentes, responsáveis pela formulação, condução e desenvolvimento da PNEPS nas locorregiões, contribuindo para o acompanhamento e avaliação das ações da política e apoiando os gestores nas discussões sobre EPS. As CIES têm em sua composição representantes do Quadrilátero da Formação: gestores, profissionais da saúde, instituições de ensino e controle social ${ }^{30}$.

Em uma avaliação recente sobre os cursos de qualificação dos trabalhadores do SUS, no estado do Ceará, demonstra-se que os fatores que podem contribuir, para a evasão dos profissionais/alunos são: a relação da extensão territorial destas macrorregiões e a centralização dos locais onde são oferecidos alguns cursos que requerem deslocamento dos profissionais, o que necessita do apoio dos gestores para transporte ou empenho pessoal para arcar com esta despesa. Outro fator que se deve considerar são os períodos de desenvolvimento dos cursos, que coincidem com o horário de trabalho dos profissionais, os mesmos necessitando então de liberação do gestor para viabilizar a sua participação nas ações, o que muitas vezes não acontece, mesmo tendo sido pactuado no Pacto pela Gestão no que diz respeito à EPS a contrapartida da gestão municipal, sendo este fator fundamental para minimizar a evasão nas ações de EPS $^{29}$.

Ainda assim, são relevantes os progressos alcançados no sentido de se efetivar a descentralização da política de saúde com os ganhos proporcionados pela adequação das ações às necessidades da população local, a capacitação de grande contingente de profissionais de atuação municipal habilitados a manejar os instrumentos do SUS, rompendo as barreiras de programas verticalizados, visando assim a melhor qualificação da prestação de serviços de saúde ${ }^{31,32}$. 
O estudo apresentou algumas limitações, uma vez que das 19 Regiões de Saúde descritas no COAP do estado, sete delas não foram avaliadas, representando 36,84\% do espaço territorial da investigação e 38,36\% da população do Estado do Ceará7. Os dados disponíveis nos documentos analisados não permitiram discutir as formas de seleção dos profissionais para as qualificações ofertadas nas regiões e nem o impacto destas qualificações na atenção à saúde ou no processo de regionalização. Desse modo, se faz importante a realização de novas investigações que superem as limitações desse estudo, propondo a capacitação da gestão para melhorar os resultados dos sistemas de saúde de nosso país.

\section{CONSIDERAÇÕES FINAIS}

O estudo ilustrou, por meio de uma análise documental, que ocorrem carências na gestão do SUS refletindo sobre a distribuição do número de profissionais por região, sobre a vinculação dos profissionais e sobre a qualificação técnica, ocasionando a precarização do serviço e dos trabalhadores, interferindo na motivação dos empregados, que evita estimular a desenvolver uma melhor assistência nas instituições do sistema.

Destarte, foi possível encontrar evidências de uma distribuição irregular das categorias profissionais nas Regiões de Saúde analisadas, segundo parâmetros apresentados em outros documentos, bem como profissionais com vínculos empregatícios fragilizados, e uma oferta de qualificação que não contemplava uma distribuição equânime por região, em decorrência de uma pactuação também irregular dos cursos ofertados deliberadas pelos colegiados de gestão regional. Portanto, propõe-se a necessidade de uma redemocratização na gestão em saúde, a fim de se efetivar os COAPs, ainda em vigência, fortalecendo a regionalização do SUS.

\section{REFERÊNCIAS BIBLIOGRÁFICAS}

1. Ministério da Saúde. Secretaria de Gestão Estratégica e Participativa. Departamento de Articulação Interfederativa. Guia para a elaboração do Contrato Organizativo da Ação Pública: construindo o COAP passo a passo. Brasília: Ministério da Saúde, 2014.

2. Cosems RJ. Seminário Estadual Contrato Organizativo das Ações Públicas de Saúde: Uma Nova Forma de implementar o Pacto pela Saúde. Rio de Janeiro (RJ): Cosems RJ, 2011.

3. Ministério da Saúde. Conselho Nacional das Secretarias Municipais de Saúde. Reflexão aos Novos Gestores Municipais de Saúde - 2013 a 2016. Brasília (DF):Ministério da Saúde, 2013.

4. Silva EC, Gomes MHA. Impasses no processo de regionalização do SUS: tramas locais. Saúde soc. 2013; 22 (4): 1106-1116.

5. Macedo NB, Albuquerque PC, Medeiros KR. O desafio da implementação da educação 
permanente na gestão da educação na saúde. Trab. educ. saúde, Rio de Janeiro. 2014; 12(2): 379401.

6. Ministério da Saúde. Conselho Nacional de Secretários de Saúde. In: ROSA, Tatiana. Ceará e Mato Grosso do Sul assinam o COAP. Revista do Conselho Nacional de Secretários de Saúde. ano II, n. 5, julho, agosto e setembro de 2012, p. 22-25.

7. Ceará. Secretaria do Planejamento e Gestão (SEPLAG). Planejamento Participativo e Regionalizado Oficinas Regionais PPA 2012 a 2015. Fortaleza-CE: SEPLAG, 2011.

8. Soares A, Santos NR. Financiamento do Sistema Único de Saúde nos governos FHC, Lula e Dilma. Saúde debate. 2014; 38(100): 18-25.

9. Biancarelli A, Cassenote A. Demografia Médica no Brasil. São Paulo: Conselho Federal de Medicina, 2011.

10. Indicadores de recursos [Internet]. Brasília (DF): Ministério da Saúde. 2011 [Citado em: abril, 2016]. Disponível em: http://tabnet.datasus.gov.br/cgi/deftohtm.exe?idb2011/e01.def.

11. Ney MS, Rodrigues PHA. Fatores críticos para a fixação do médico na Estratégia Saúde da Família. Physis. 2012; 22( 4 ): 1293-1311.

12. Gonçalves CR et al. Recursos humanos: fator crítico para as redes de atenção à saúde. Saúde debate. 2014; 38(100): 26-34.

13. Baratieri T, Mandu ENT, Marcon SS. Longitudinalidade no trabalho do enfermeiro: relatos da experiência profissional. Rev. esc. enferm. USP. 2012; 46(5): 1260-1267.

14. Cunha EM da, Giovanella L. Longitudinalidade/continuidade do cuidado: identificando dimensões e variáveis para a avaliação da Atenção Primária no contexto do sistema público de saúde brasileiro. Ciênc. saúde coletiva. 2011; 16 (Suppl 1): 1029-1042.

15. Baratieri T, Marcon SS. Longitudinalidade do cuidado: compreensão dos enfermeiros que atuam na estratégia saúde da família. Esc. Anna Nery. 2011; 15(4): 802-810.

16. Verde FF, Bernardo MH. Vivências de trabalhadores com diferentes vínculos empregatícios em um laboratório público. Anais do XVI Encontro de Iniciação Científica e I Encontro de Iniciação em Desenvolvimento Tecnológico e Inovação da PUC-Campinas, 27 e 28 de setembro de 2011.

17. Gonçalves RC. O assédio moral no Ceará: naturalização dos atos injustos no trabalho [Dissertação]. Fortaleza-Ce: UNIFOR, 2006. 109 p. 
18. Cavalcante MVS, Lima TCS. A precarização do trabalho na atenção básica em saúde: relato de experiência. Argumentum. 2013; 5(1): 235-256.

19. Oliveira AKL. Assédio moral no cenário do trabalho flexível: desafios da vigilância em saúde do trabalhador no Ceará [Dissertação]. Fortaleza-Ce: UECE, 2013.

20. Silva IV, Aquino EM L, Pinto ICM. Violência no trabalho em saúde: a experiência de servidores estaduais da saúde no Estado da Bahia, Brasil. Cad. Saúde Pública. 2014; 30(10): 2112-2122.

21. Jorge MSB, Guimarães JMX, Nogueira MEF, Moreira TMM, Morais APP. Gestão de recursos humanos nos centros de atenção psicossocial no contexto da Política de Desprecarização do Trabalho no Sistema Único de Saúde. Texto contexto-enferm. 2007; 16 (3): 417-425.

22. Souza GCA, Costa ICC. O SUS nos seus 20 anos: reflexões num contexto de mudanças. Saúde soc. 2010; 19(3): 509-517.

23. Ministério da Saúde. Secretaria de Gestão do Trabalho e da Educação na Saúde. Departamento de Gestão e da Regulação do Trabalho em Saúde. Programa Nacional de Desprecarização do Trabalho no SUS: DesprecarizaSUS - perguntas \& respostas. Brasília: Editora do Ministério da Saúde, 2006.

24. Mendes EV. O cuidado das condições crônicas na atenção primária à saúde: o imperativo da consolidação da estratégia da saúde da família. Brasília: Organização Pan-Americana da Saúde, 2012.

25. Barth PO. Educação permanente em saúde: concepções e práticas em centros de saúde de Florianópolis [Dissertação]. Florianópolis (SC): UFSC, 2012.

26. Stroschein, KA, Zocche DAA. Educação permanente nos serviços de saúde: um estudo sobre as experiências realizadas no Brasil.Trab. educ. saúde. 2011; 9(3): 505-519.

27. Ceccim RB. Educação Permanente em Saúde: descentralização e disseminação de capacidade pedagógica na saúde. Ciênc. Saúde Coletiva. 2005; 10(4): 975-986.

28. Vieira M, Chinelli F. The contemporary relationship between work, qualification and recognition: repercussions on the Unified Health System (SUS) workers. Ciênc. saúde coletiva. 2013; 18(6): 1591-1600.

29. Chagas MIO, Costa AA, Machado MFAS, Dias MAS, Teófilo KS, Cavalcante ASP. Implantação da Política Nacional de Educação Permanente em Saúde no Estado do Ceará: Um Estudo de Avaliabilidade. Gaceta Sanitaria (Barcelona. Ed. impresa). 2013; 27(4): 324-325. 
$93 / /$

30. Ministério da Saúde. Secretaria de Gestão do Trabalho e da Educação na Saúde. Departamento de Gestão da educação em Saúde. Política Nacional de Educação Permanente em Saúde. Brasília: Ministério da Saúde, 2009.

31. Duarte Ligia Schiavon, Pessoto Umberto Catarino, Guimarães Raul Borges, Heimann Luiza Sterman, Carvalheiro José da Rocha, Cortizo Carlos Tato et al . Regionalização da saúde no Brasil: uma perspectiva de análise. Saude soc. 2015 June; 24( 2 ): 472-485.

32. Rockers PC, Bärnighausen T. Interventions for hiring, retaining and training district health systems managers in low- and middle-income countries. Cochrane Database of Systematic Reviews. 2013; 1(4): 5-6.

Artigo apresentado em 16-01-15

Artigo aprovado em 10-01-16 Artigo publicado no sistema em 23-03-16 